\title{
Clinical Impact of the Introduction of an Early Rehabilitation Protocol on Infectious Complications in Patients after Gastrointestinal Cancer Surgery
}

\author{
Yasuhisa Fukawa, RPT a Wataru Kakuda, MD, PhD ${ }^{\mathrm{b}}$ Seiva Yoshida, RPT a Masashi Zenta RPT a \\ Osamu Itano, MD, PhD ${ }^{\mathrm{c}}$ Risa Kiko, $\mathrm{ST}^{\mathrm{a}}$ Hiroaki Tani, RPT, PhD ${ }^{\mathrm{d}}$ and Akira Kubo, RPT, PhD ${ }^{\mathrm{d}}$
}

\begin{abstract}
Background: The clinical importance of postoperative rehabilitation for cancer patients has recently attracted much attention. However, it remains uncertain whether early rehabilitation can prevent infectious complications in patients undergoing gastrointestinal cancer surgery. Methods: The study group consisted of 259 patients who underwent laparoscopic or open surgery for gastrointestinal cancer at our institution between December 2012 and November 2016. Our department proposed a new early rehabilitation protocol for such patients to encourage physical activity after surgery. The protocol was clinically introduced on July 21,2015 . We divided the study subjects into two groups: those who were admitted before the introduction of the new protocol and those admitted after. The frequency of infectious complications, including respiratory infections, and the length of hospital stay after surgery were compared between the two groups. Results: No adverse cardiovascular event associated with the early rehabilitation protocol was experienced. After the protocol was introduced, more than $80 \%$ of patients started exercising on the first day after surgery. For patients undergoing open surgery, the frequency of infectious complications was significantly reduced with the introduction of the protocol $(\mathrm{p}<0.05)$. Moreover, when open surgery was performed, the protocol significantly shortened the length of hospital stay $(p<0.05)$. Conclusion: Our proposed early rehabilitation protocol for patients who have undergone gastrointestinal cancer surgery was considered to be safe and feasible. The protocol may prevent infectious complications and shorten the hospital stay after such surgery.
\end{abstract}

Key words: cancer surgery; early rehabilitation; gastrointestinal cancer; infectious complication; postoperative rehabilitation

\section{INTRODUCTION}

Surgery is an important treatment modality for gastrointestinal cancers such as gastric and colon cancer. Progress in surgical techniques in recent decades has noticeably reduced operative mortality rates in patients undergoing such surgery. ${ }^{1,2)}$ However, complications associated with gastrointestinal cancer surgery are still fairly common. Infectious complications, including pneumonia and wound infections, are important and are frequently encountered in the clinical setting. ${ }^{3,4)}$ The development of these infectious complications can negatively affect the clinical outcomes of patients undergoing gastrointestinal cancer surgery. For example, the length of hospital stay after gastrointestinal cancer surgery can be prolonged with the development of such complications, leading to an increased financial burden of these cancers. ${ }^{5,6)}$ Effective interventions to minimize or even prevent infectious complications need to be provided as soon as possible after surgery.

Recently, the clinical importance of cancer rehabilitation

Received: August 23, 2018, Accepted: January 27, 2019, Published online: February 6, 2019

a Department of Rehabilitation Medicine, International University of Health and Welfare Ichikawa Hospital, Ichikawa, Chiba, Japan

${ }^{\mathrm{b}}$ Department of Rehabilitation Medicine, International University of Health and Welfare School of Medicine, Narita, Chiba, Japan

${ }^{c}$ Department of Hepato-Biliary-Pancreatic Gastrointestinal Surgery, International University of Health and Welfare School of Medicine, Narita, Chiba, Japan

${ }^{d}$ Department of Physical Therapy, Faculty of Health Science, International University of Health and Welfare, Otawara, Tochigi, Japan Correspondence: Prof. Wataru Kakuda, MD, PhD, Department of Rehabilitation Medicine, School of Medicine, International University of Health and Welfare, 4-3 Kozunomori, Narita, Chiba 286-8686, Japan, Email: wkakuda@iuhw.ac.jp

Copyright (C) 2019 The Japanese Association of Rehabilitation Medicine 
has been attracting much attention, not only in the field of rehabilitation medicine, but also in oncology. ${ }^{7,8)}$ The goal of cancer rehabilitation is generally to improve daily physical function and quality of life for patients living with cancer. In particular, postoperative rehabilitation for cancer patients is important. The number of gastrointestinal cancer patients undergoing early rehabilitation after surgery has been increasing. ${ }^{9,10)}$ However, the influence of early rehabilitation for patients undergoing gastrointestinal cancer surgery on the development of infectious complications remains unknown. It is hoped that the provision of early rehabilitation could lead to a reduction in the rate of infectious complications in such patients. The aim of this study was to assess the influence of early rehabilitative exercise on infectious complications in patients undergoing gastrointestinal cancer surgery. Furthermore, we attempted to identify any differences in the efficacy of rehabilitative exercise between patients undergoing laparoscopic surgery and open surgery for gastrointestinal cancer.

\section{SUBJECTS AND METHODS}

The study protocol was approved by the ethics committee of our institution (International University of Health and Welfare Ichikawa Hospital). This study was carried out in compliance with the Declaration of Helsinki. It involved a retrospective analysis of a prospectively enrolled consecutive cohort of patients who underwent gastrointestinal cancer surgery. All patients underwent surgical treatment for gastrointestinal cancer at our institution between December 1, 2012, and November 30, 2016. Both patients who underwent laparoscopic surgery and those who underwent open surgery were included in this study. Patients were excluded if the surgery was performed emergently or if the patient needed artificial ventilation with endotracheal intubation for several days after the surgery.

To encourage physical activity and to prevent the development of disuse syndrome after surgery, a new early rehabilitation protocol after gastrointestinal cancer surgery was proposed at our institution and was clinically introduced for patients on July 21, 2015. Before the protocol was introduced, surgeons at the Department of Surgery and rehabilitation physicians and physical therapists at the Department of Rehabilitation Medicine at our institution had several meetings to discuss the clinical concept of the protocol and to standardize the exercises provided in the protocol. Although we basically aimed to initiate rehabilitative exercise at the bedside on the first day after surgery, the decision to initiate early rehabilitation was carefully determined by rehabilitation physicians or physical therapists. In cases where consciousness was still disturbed (the patient could not open their eyes), a high fever $\left(>38.0^{\circ} \mathrm{C}\right)$ persisted, or artificial ventilation was needed, the initiation of the protocol was delayed and exercise was not started until the patient's condition had improved. The exercises in the protocol included range-ofmotion exercises, muscle strengthening exercises for the lower limb muscles, sitting training, standing training, gait training, and respiratory rehabilitation. Respiratory rehabilitation involved training to loosen secretions, to produce effective coughing, and to strengthen respiratory muscles. The duration of the exercises varied between 20 and $40 \mathrm{~min} /$ day. Essentially, exercises in the protocol were provided daily until patient discharge; however, the content of the exercise was varied in accordance with the recovery of physical functions. Throughout each exercise session, the development of adverse cardiovascular events was continuously monitored by physical therapists.

In this study, the subjects were divided into two groups: the pre-introduction group, who were admitted before the introduction of the protocol, and the post-introduction group, who were admitted after the introduction. The frequency of infectious complications that developed within 1 month after surgery and the length of hospital stay after surgery were compared between the two groups for patients who underwent laparoscopic and open surgery. The following clinical data were collected retrospectively from the medical records: age at admission, gender, diagnosis, clinical stage of the cancer, type of surgery (laparoscopic or open surgery), surgery duration (from the initial skin incision to the completion of suturing), amount of bleeding during surgery, comorbidities (hypertension, diabetes, cardiac diseases, respiratory diseases, and renal diseases), baseline ADL level, and body mass index at admission.

The development of infectious complications, such as respiratory infections, wound infections, and systemic infections, was confirmed based on the medical records. For example, a diagnosis of respiratory infection was made in cases where the amount of sputum increased and abnormal shadows were noted on chest X-rays. It was considered that wound infection had developed when skin redness around the wound persisted longer than usual or abscess formation was found on computed tomography scans of the abdomen. Systemic infection refers to sepsis and systemic inflammatory response syndrome. The diagnosis of these systemic infections was based on the published diagnostic criteria for each condition. ${ }^{11,12)}$ 
All statistical analyses were performed using SPSS version 23.0 (SPSS Inc, Chicago, Il, USA). Comparison of the clinical characteristics (i.e., age, surgery duration, amount of bleeding, and body mass index) between the two groups (the pre-introduction group and the post-introduction group) was carried out using the unpaired Student's $t$-test for continuous variables. Categorical variables (i.e., gender, diagnosis, stage, type of surgery, comorbidities, and ADL level) were compared using the chi-square test. The effect of the timing of rehabilitation initiation was analyzed using the chi-square test. The frequency of infectious complications was analyzed for each complication (respiratory infection, wound infection, and systemic infection) using the chi-square test. Analysis of changes in the length of hospital stay with the introduction of the protocol was carried out using the unpaired Student's $t$-test. A p-value of less than 0.05 was considered significant.
The design of this study was in accordance with the ethical standards of the institutional and national research committee and with the 1964 Helsinki declaration and its later amendments. This article does not contain any procedures on animals performed by any of the authors.

\section{RESULTS}

In total, 259 patients who underwent gastrointestinal cancer surgery were included in this study. Of these, 124 patients (laparoscopic surgery, 91; open surgery, 33) belonged to the pre-introduction group, and 135 patients (laparoscopic surgery, 99; open surgery, 36) belonged to the post-introduction group. The baseline clinical characteristics of the two groups are shown in Table 1; there were no significant differences between the groups, except for the duration of surgery in the

Table 1. Baseline clinical characteristics of the patients studied

\begin{tabular}{|c|c|c|c|c|}
\hline \multicolumn{2}{|c|}{ Characteristics } & $\begin{array}{l}\text { Pre-introduction group } \\
\qquad(\mathrm{n}=124)\end{array}$ & \multirow{2}{*}{$\begin{array}{c}\begin{array}{c}\text { Post-introduction group } \\
(\mathrm{n}=135)\end{array} \\
68.5 \pm 10.6\end{array}$} & \multirow{2}{*}{$\begin{array}{l}\text { P value } \\
\text { n.s. }\end{array}$} \\
\hline \multirow{4}{*}{ Age at admission } & All patients (years) & $68.2 \pm 10.2$ & & \\
\hline & $<60$ (years) & $22(18)$ & $25(19)$ & \multirow{3}{*}{ n.s. } \\
\hline & $60-70$ (years) & $48(39)$ & $49(36)$ & \\
\hline & $>70$ (years) & $54(43)$ & $61(45)$ & \\
\hline \multirow{2}{*}{ Gender } & Female & $52(42)$ & $65(48)$ & \multirow{2}{*}{ n.s. } \\
\hline & Male & $72(58)$ & $70(52)$ & \\
\hline \multirow{3}{*}{ Diagnosis } & Gastric cancer & $33(27)$ & $52(39)$ & \multirow{3}{*}{ n.s. } \\
\hline & Colon cancer & $63(51)$ & $60(44)$ & \\
\hline & Rectal cancer & $28(22)$ & $23(17)$ & \\
\hline \multirow{4}{*}{ Clinical stage of the cancer } & 1 & $43(35)$ & $57(42)$ & \multirow{4}{*}{ n.s. } \\
\hline & 2 & $36(29)$ & $24(18)$ & \\
\hline & 3 & $31(25)$ & $34(25)$ & \\
\hline & 4 & $14(11)$ & $20(15)$ & \\
\hline \multirow{2}{*}{ Type of surgery } & Laparoscopic surgery & $91(73)$ & $99(73)$ & \multirow{2}{*}{ n.s. } \\
\hline & Open surgery & $33(27)$ & $36(27)$ & \\
\hline \multirow{2}{*}{ Surgery duration (min) } & Laparoscopic surgery & $94 \pm 29$ & $113 \pm 49$ & $<0.05$ \\
\hline & Open surgery & $134 \pm 56$ & $153 \pm 67$ & n.s. \\
\hline \multirow{2}{*}{$\begin{array}{l}\text { Amount of bleeding during } \\
\text { surgery }(\mathrm{ml})\end{array}$} & Laparoscopic surgery & $118 \pm 262$ & $75 \pm 140$ & \multirow[b]{2}{*}{ n.s. } \\
\hline & Open surgery & $435 \pm 893$ & $254 \pm 271$ & \\
\hline \multirow{5}{*}{ Comorbidities } & Hypertension & $21(17)$ & $23(17)$ & n.s. \\
\hline & Diabetes & $65(52)$ & $79(59)$ & n.s. \\
\hline & Cardiac diseases & $46(37)$ & 39 (29) & n.s. \\
\hline & Respiratory diseases & $22(18)$ & $15(11)$ & n.s. \\
\hline & Renal diseases & $6(5)$ & $6(4)$ & n.s. \\
\hline \multirow{2}{*}{ Baseline ADL level } & Independent & $117(94)$ & $126(93)$ & \multirow{2}{*}{ n.s. } \\
\hline & Dependent (partially or totally) & $7(6)$ & $9(7)$ & \\
\hline \multicolumn{2}{|c|}{ Body mass index at admission $\left(\mathrm{kg} / \mathrm{m}^{2}\right)$} & $24.1 \pm 5.1$ & $22.9 \pm 3.9$ & n.s. \\
\hline
\end{tabular}

Values are numbers $(\%)$ or mean \pm standard deviation. n.s., not significant. 


\section{Pre-introduction Group $(n=124)$}

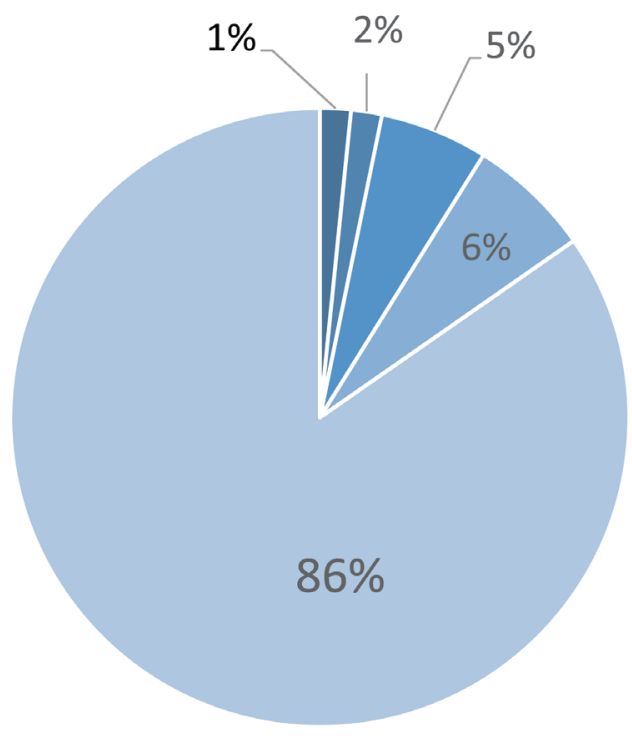

Fig. 1. Timing of rehabilitation initiation after surgery.

\section{Post-introduction Group $(n=135)$}

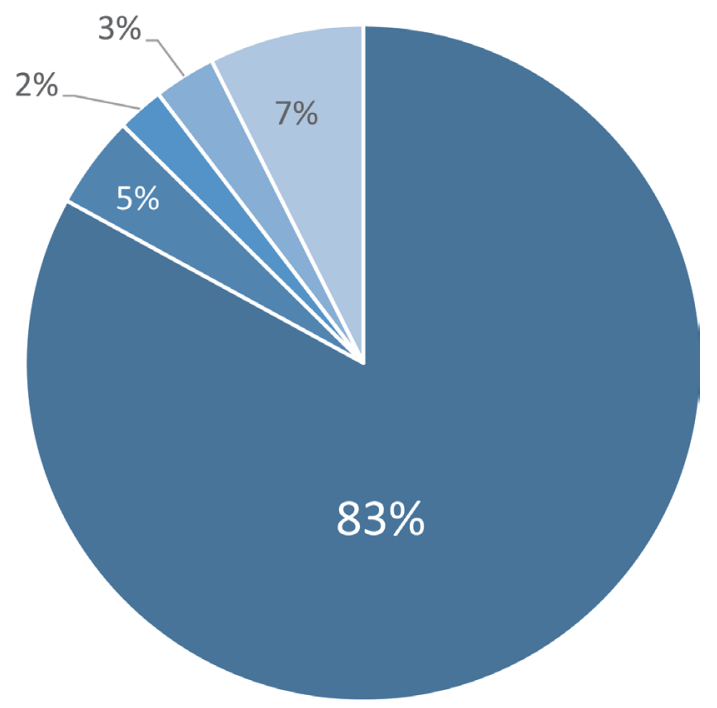

laparoscopic surgery patients. Although the duration of surgery was significantly longer in the post-introduction group than in the pre-introduction group $(\mathrm{p}<0.05)$, the difference between two groups was relatively small (about $20 \mathrm{~min}$ ). As shown in Fig. 1, the timing of rehabilitation initiation after surgery significantly differed between the two groups $(\mathrm{p}<0.05)$. In the pre-introduction group, only $8 \%$ of patients started rehabilitative exercise during the first seven days after surgery. For $86 \%$ of patients in the pre-introduction group, no exercise was provided after surgery. In contrast, in the post-introduction group, $83 \%$ of patients started exercise on the first day after surgery. Furthermore, exercise was initiated during the first seven days in $90 \%$ of patients in the post-introduction group. No adverse cardiovascular event associated with initiation of the early rehabilitation protocol, e.g., cerebral ischemic symptoms or deterioration of cardiac function, was observed. On the introduction of the early rehabilitation protocol, some changes in the frequency of infectious complications were found, as shown in Fig. 2. After laparoscopic surgery, changes in the frequency of infectious complications with the introduction of the protocol were not statistically significant (total infections: from $24.2 \%$ to $18.2 \%, \mathrm{p}=0.31$; respiratory infections: from $5.5 \%$ to $5.1 \%, p=0.89$; wound infections: from $19.8 \%$ to $11.1 \%$, $\mathrm{p}=0.09$; and systemic infections: from $1.1 \%$ to $4.0 \%, \mathrm{p}=0.21$ ). However, after open surgery, introduction of the protocol was significantly beneficial for the prevention of infectious complications. In particular, the frequencies of respiratory and systemic infections were significantly decreased with the introduction of the protocol (total infections: from $45.5 \%$ to $22.2 \%, \mathrm{p}<0.05$; respiratory infections: from $24.2 \%$ to $2.8 \%$, $\mathrm{p}<0.05$; wound infections: from $27.3 \%$ to $19.4 \%, \mathrm{p}=0.44$; and systemic infections: from $12.1 \%$ to $0.0 \%, \mathrm{p}<0.05)$. In addition, as shown in Fig. 3, the introduction of the protocol shortened the length of hospital stay after laparoscopic surgery, although this was not statistically significant (from $22.0 \pm 17.5$ to $18.7 \pm 9.3$ days, $p=0.054)$. However, the length of hospital stay after open surgery was significantly shortened with the protocol (from $39.1 \pm 26.7$ to $27.3 \pm 14.8$ days, $\mathrm{p}<0.05$ ). 


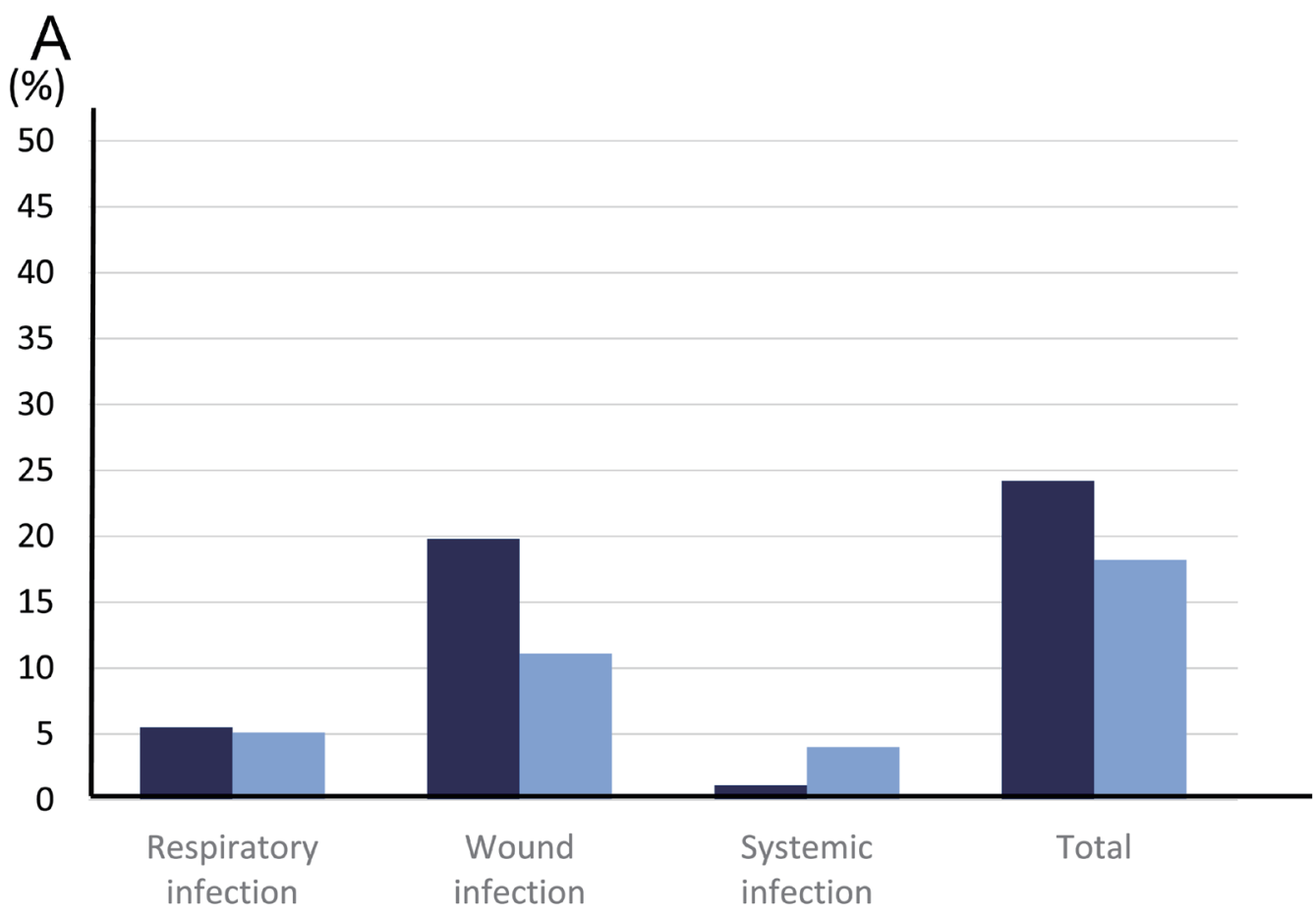

- Pre-introduction Group a Post-introduction Group

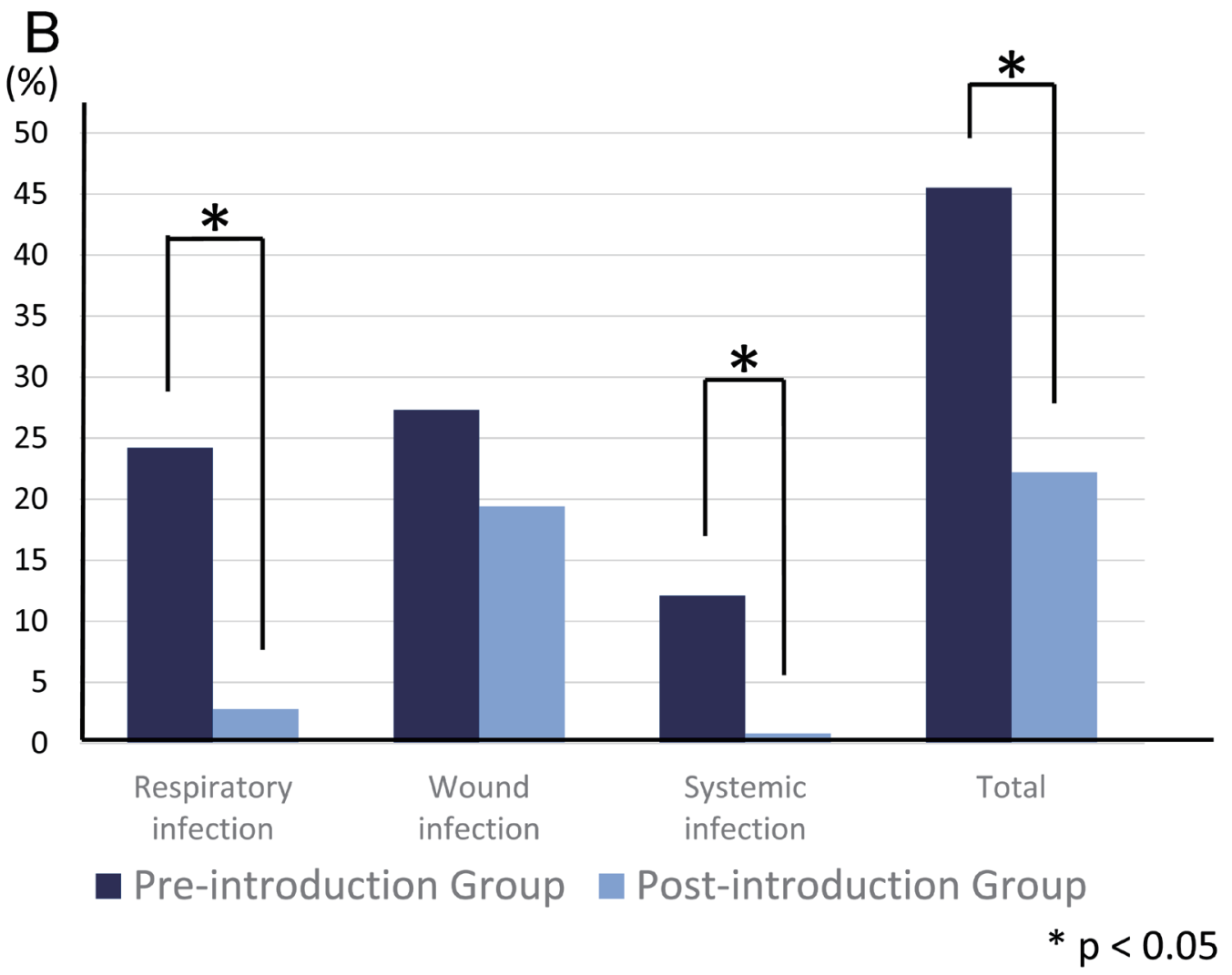

Fig. 2. Frequency of infectious complications after surgery. (A) Patients after laparoscopic surgery, (B) patients after open surgery. 

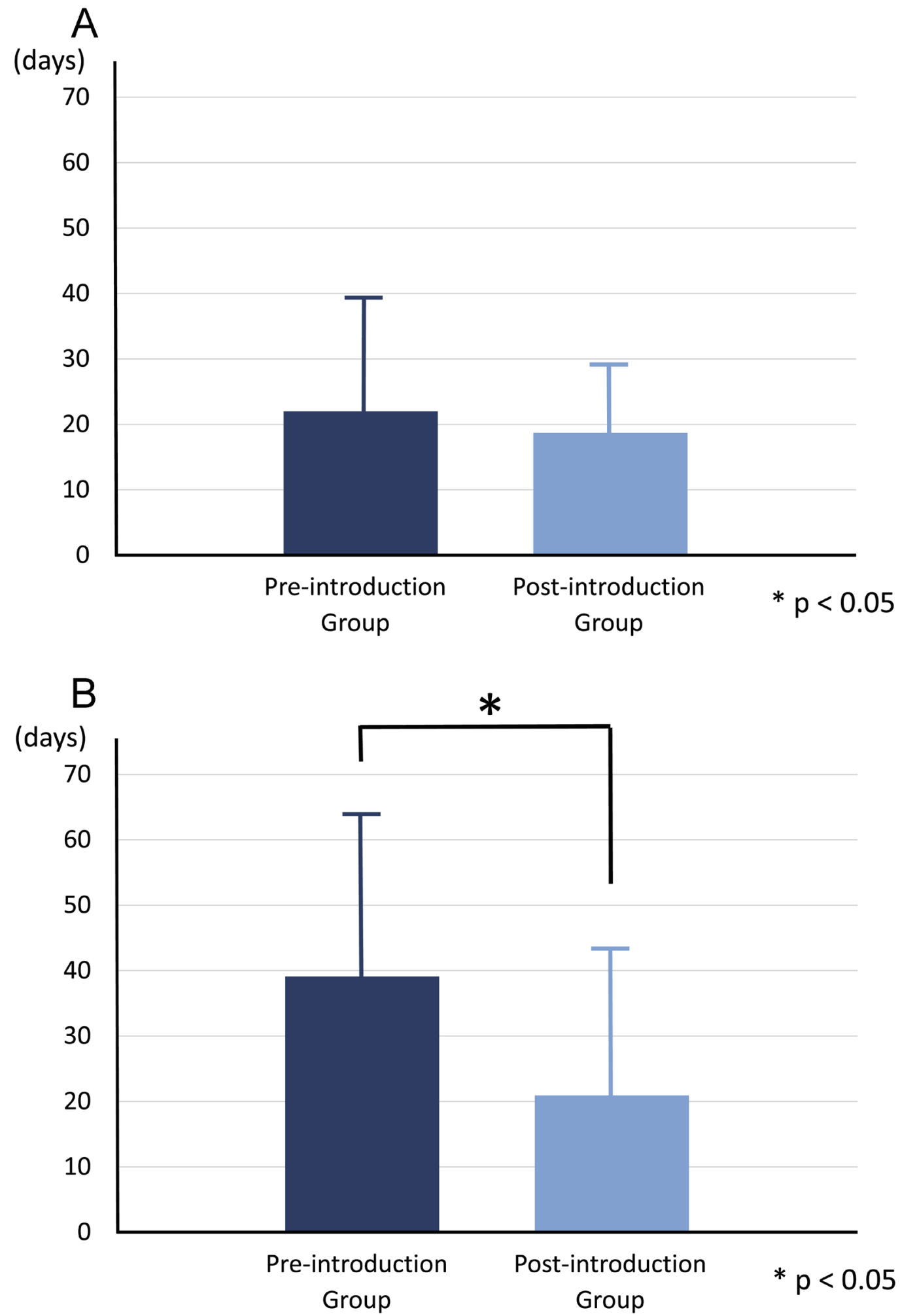

Fig. 3. Length of hospital stay after surgery. (A) Patients after laparoscopic surgery, (B) patients after open surgery. 


\section{DISCUSSION}

We proposed an early rehabilitation protocol for patients undergoing gastrointestinal cancer surgery and we successfully and safely introduced the protocol for these patients. Our findings indicate that the protocol can be considered a safe and feasible rehabilitative intervention for patients after gastrointestinal cancer surgery. The early intervention protocol decreased the development of infectious complications after gastrointestinal cancer surgery, especially when open surgery was performed. Furthermore, the length of hospital stay after surgery was shortened after the protocol was introduced.

With the introduction of our proposed protocol, the time between surgery and the initiation of postoperative rehabilitation was greatly shortened. Some clinicians may be concerned about the development of adverse events when postoperative rehabilitation is initiated early. ${ }^{13)}$ In our patients, however, no adverse event was found with the initiation of our proposed protocol. For our patients, whether early rehabilitation could be initiated was carefully considered based on the postoperative condition of each patient. If a patient was in a critical condition after surgery, the initiation of rehabilitation was delayed. This exclusion from early postoperative rehabilitation may have contributed to the safety of our proposed protocol.

The results of this study indicated that our proposed early rehabilitation protocol for post-surgery patients is significantly beneficial when open surgery is performed. Although the underlying mechanism for the decrease of infectious complications after open surgery with our proposed protocol remains to be investigated, we can speculate about some mechanisms for the decrease. After open surgery for gastrointestinal cancer, some physiological changes can occur that are considered to lead to the development of infectious complications; these changes include a decline in pulmonary function, the release of inflammatory mediators, and nutritional deficiency. Importantly, these changes can be potentiated by prolonged bed rest. ${ }^{14)}$ In our proposed protocol, early mobilization and respiratory rehabilitation was aggressively introduced to prevent immobility and maintain pulmonary function after surgery. The protocol may have been beneficial in preventing such harmful physiological changes. ${ }^{15}$ ) Furthermore, some researchers have studied the influence of rehabilitative exercise on immune systems. Na et al. reported that early moderate exercise has a beneficial effect on the cytotoxic function of natural killer cells in stomach cancer patients after curative surgery. ${ }^{16)}$ In the report by Allgayer et al., short-term moderate exercise in patients after colorectal cancer surgery was shown to reduce oxidative DNA damage as a consequence of its favorable effect on the immune system. ${ }^{17)}$ In contrast, some articles demonstrating that bed rest without exercise can suppress immune functions have been published. ${ }^{18,19)}$ Therefore, it can be speculated that rehabilitative exercise provided in our proposed protocol may have favorably influenced patients' immune systems, leading to the suppression of infectious complications.

Our proposed protocol also significantly shortened the length of hospital stay in patients undergoing open surgery for gastrointestinal cancer. Previously, some researchers have reported that the length of hospital stay after surgery can be influenced by postoperative complications. Collins et al. assessed the association of some clinical variables with prolonged length of hospital stay in patients undergoing major elective surgery. ${ }^{5}$ ) They found that the development of postoperative complications, such as pneumonia, is significantly associated with a prolonged length of hospital stay. Similarly, Ricciardi et al. performed a retrospective analysis to identify variables predictive of prolonged length of hospital stay in patients after colorectal surgery. ${ }^{20)}$ The results of their multivariate analysis revealed that the presence of postoperative complications, including pneumonia, sepsis, and wound infection, was predictive of a prolonged length of hospital stay. Therefore, it is considered that the shortening of the hospital stay after open surgery in the current study may be caused partly by the prevention of infectious complications with the introduction of early postoperative rehabilitation. This shortening of the hospital stay with our proposed protocol can contribute to the reduction of medical cost for patients undergoing open surgery for gastrointestinal cancer. However, the cost-effectiveness of our proposed protocol remains unknown. It should be taken into consideration and investigated thoroughly in the future.

The present study has some limitations. First, this is a retrospective analysis comparing clinical outcomes before and after the introduction of a new protocol. Although it is considered that, based on the results of this study, the safety and feasibility of the protocol was confirmed, the beneficial efficacy of the protocol should be validated in studies of a randomized controlled trial design with a control group. Second, data regarding pre-operative physical fitness levels, such as cardiac and pulmonary function, were not available for the studied patients. These clinical features might influence the development of postoperative infectious complications, especially respiratory infection. The influence of preoperative physical fitness on patient outcomes is also worthy 
of study. Third, the influence of the protocol on functional recovery was not sufficiently assessed in this study, although the protocol proved to significantly shorten the length of hospital stay after surgery. It remains to be investigated whether the introduction of the protocol can accelerate the recovery of functions such as walking and muscle power in the lower limbs.

\section{CONCLUSION}

At our department, a new early rehabilitation protocol was successfully and safely introduced for patients undergoing gastrointestinal cancer surgery. For patients who underwent open surgery, the introduction of the protocol significantly decreased the frequency of infectious complications after surgery and also significantly shortened the length of hospital stay. We consider that our early rehabilitation protocol may be beneficial for the prevention of infectious complications after gastrointestinal cancer surgery. It is hoped that the protocol will be introduced more widely to further promote postoperative rehabilitation.

\section{ACKNOWLEDGMENT}

The authors gratefully acknowledge the support and the participation of the patients in this study.

\section{CONFLICT OF INTEREST}

The authors declare no conflict of interest.

\section{REFERENCES}

1. Siegel RL, Miller KD, Jemal A: Cancer statistics, 2015. CA Cancer J Clin 2015;65:5-29. PMID:25559415, DOI:10.3322/caac. 21254

2. La Vecchia C, Rota M, Malvezzi M, Negri E: Potential for improvement in cancer management: reducing mortality in the European Union. Oncologist 2015;20:495-498. PMID:25888268, DOI:10.1634/theoncologist.2015-0011

3. Jiang SP, Li ZY, Huang LW, Zhang W, Lu ZQ, Zheng $\mathrm{ZY}$ : Multivariate analysis of the risk for pulmonary complication after gastrointestinal surgery. World J Gastroenterol 2005;11:3735-3741. PMID:15968730, DOI:10.3748/wjg.v11.i24.3735
4. Rovera F, Dionigi G, Boni L, Piscopo C, Masciocchi P, Alberio MG, Carcano G, Diurni M, Dionigi R: Infectious complications in colorectal surgery. Surg Oncol 2007;16(Suppl 1):121-124. PMID:18032026, DOI:10.1016/j.suronc.2007.10.029

5. Collins TC, Daley J, Henderson WH, Khuri SF: Risk factors for prolonged length of stay after major elective surgery. Ann Surg 1999;230:251-259. PMID:10450740, DOI:10.1097/00000658-199908000-00016

6. Dimick JB, Chen SL, Taheri PA, Henderson WG, Khuri SF, Campbell DA Jr: Hospital costs associated with surgical complications: a report from the private-sector National Surgical Quality Improvement Program. J Am Coll Surg 2004;199:531-537. PMID:15454134, DOI:10.1016/j.jamcollsurg.2004.05.276

7. Spence RR, Heesch KC, Brown WJ: Exercise and cancer rehabilitation: a systematic review. Cancer Treat Rev 2010;36:185-194. PMID:19962830, DOI:10.1016/j. ctrv.2009.11.003

8. Egan MY, McEwen S, Sikora L, Chasen M, Fitch M, Eldred S: Rehabilitation following cancer treatment. Disabil Rehabil 2013;35:2245-2258. PMID:23488617, DOI:10.3109/09638288.2013.774441

9. Wang G, Jiang ZW, Xu J, Gong JF, Bao Y, Xie LF, Li JS: Fast-track rehabilitation program vs conventional care after colorectal resection: a randomized clinical trial. World J Gastroenterol 2011;17:671-676. PMID:21350719, DOI:10.3748/wjg.v17.i5.671

10. Wong CL, Lee HH, Chang SC: Colorectal cancer rehabilitation review. J Cancer Res Pract 2016;3:31-33. DOI:10.1016/j.jcrpr.2015.07.001

11. Singer M, Deutschman CS, Seymour CW, ShankarHari M, Annane D, Bauer M, Bellomo R, Bernard GR, Chiche JD, Coopersmith CM, Hotchkiss RS, Levy MM, Marshall JC, Martin GS, Opal SM, Rubenfeld GD, van der Poll T, Vincent JL, Angus DC: The third international consensus definitions for sepsis and septic shock (Sepsis-3). JAMA 2016;315:801-810. PMID:26903338, DOI:10.1001/jama.2016.0287

12. Kaukonen KM, Bailey M, Pilcher D, Cooper DJ, Bellomo R: Systemic inflammatory response syndrome criteria in defining severe sepsis. N Engl J Med 2015;372:1629-1638. PMID:25776936, DOI:10.1056/ NEJMoa1415236 
13. Launay-Savary MV, Mathonnet M, Theissen A, Ostermann S, Raynaud-Simon A, Slim K, GRACE (Groupe francophone de Réhabilitation Améliorée après Chirurgie): Are enhanced recovery programs in colorectal surgery feasible and useful in the elderly? A systematic review of the literature. J Visc Surg 2017;154:29-35. PMID:27842907, DOI:10.1016/j.jviscsurg.2016.09.016

14. Havey R, Herriman E, O'Brien D: Guarding the gut: early mobility after abdominal surgery. Crit Care Nurs Q 2013;36:63-72. PMID:23221443, DOI:10.1097/ CNQ.0b013e3182753237

15. Castelino T, Fiore JF Jr, Niculiseanu P, Landry T, Augustin B, Feldman LS: The effect of early mobilization protocols on postoperative outcomes following abdominal and thoracic surgery: a systematic review. Surgery 2016;159:991-1003. PMID:26804821, DOI:10.1016/j. surg.2015.11.029

16. Na YM, Kim MY, Kim YK, Ha YR, Yoon DS: Exercise therapy effect on natural killer cell cytotoxic activity in stomach cancer patients after curative surgery. Arch Phys Med Rehabil 2000;81:777-779. PMID:10857523, DOI:10.1016/S0003-9993(00)90110-2
17. Allgayer H, Owen RW, Nair J, Spiegelhalder B, Streit J, Reichel C, Bartsch H: Short-term moderate exercise programs reduce oxidative DNA damage as determined by high-performance liquid chromatography-electrospray ionization-mass spectrometry in patients with colorectal carcinoma following primary treatment. Scand J Gastroenterol 2008;43:971-978. PMID:18609189, DOI:10.1080/00365520701766111

18. Murdaca G, Setti M, Brenci S, Fenoglio D, Lantieri P, Indiveri F, Puppo F: Modifications of immunological and neuro-endocrine parameters induced by antiorthostatic bed-rest in human healthy volunteers. Minerva Med 2003;94:363-378. PMID:14976465

19. Hoff P, Belavý DL, Huscher D, Lang A, Hahne M, Kuhlmey AK, Maschmeyer P, Armbrecht G, Fitzner R, Perschel FH, Gaber T, Burmester GR, Straub RH, Felsenberg D, Buttgereit F: Effects of 60-day bed rest with and without exercise on cellular and humoral immunological parameters. Cell Mol Immunol 2015;12:483-492. PMID:25382740, DOI:10.1038/ cmi.2014.106

20. Ricciardi R, Roberts PL, Read TE, Hall JF, Marcello PW, Schoetz DJ: Which adverse events are associated with mortality and prolonged length of stay following colorectal surgery? J Gastrointest Surg 2013;17:14851493. PMID:23690207, DOI:10.1007/s11605-013-2224-3 